\title{
Condensation of Vortex-Strings: Effective Potential Contribution Through Dual Actions
}

\author{
Rudnei O. Ramos, Daniel G. Barci, Cesar A. Linhares, \\ Departamento de Física Teórica, Universidade do Estado do Rio de Janeiro, 20550-013 Rio de Janeiro, RJ, Brazil
}

and J. F. Medeiros Neto

Instituto de Física, Universidade Federal do Pará, 66075-110 Belem, Pará, Brazil

(Received on 01 September, 2006)

\begin{abstract}
Topological excitations are believed to play an important role in different areas of physics. For example, one case of topical interest is the use of dual models of quantum cromodynamics to understand properties of the vacuum and confinement through the condensation of magnetic monopoles and vortices. Other applications are related to the role of these topological excitations, nonhomogeneous solutions of the field equations, in phase transitions associated to spontaneous symmetry breaking in gauge theories, whose study is of importance, for instance, in phase transitions in the early universe. Here we show a derivation of a model dual to the scalar Abelian Higgs model where its topological excitations, namely vortex-strings, become manifest and can be treated in a quantum field theory way. The derivation of the nontrivial contribution of these vacuum excitations to phase transitions and its analogy with superconductivity is then made possible and they are studied here.
\end{abstract}

Keywords: Dual models; Vortices; Phase transitions

\section{INTRODUCTION}

Topological excitations, or defects, are nonhomogeneous solutions of the field equations of motion in many types of field theory models [1-3]. They are finite energy and stable configurations that emerge as a consequence of a spontaneous symmetry breaking process. Mathematically, defects are predicted to appear whenever some larger group of symmetry $G$ breaks into a smaller one $H$ such that there are a nontrivial homotopy group $\pi_{k}(G / H)$ of the vacuum manifold different from the identity. Well known examples are kinks, or domain walls $(k=0)$ [2], that originate from a discrete symmetry breaking, strings, or vortices $(k=1)$, for example originating from a continuous gauge symmetry breaking $U(1) \rightarrow 1$ [4] and magnetic monopoles $(k=2)$, e.g. from a $S O(3) \rightarrow U(1)$ symmetry breaking $[5,6]$. Since many phase transitions in nature are associated to symmetry breakings, topological excitations are a common feature in these processes and are in fact observed in many systems in the laboratory, like in ferromagnetism, helium superfluidity, superconductivity and in many other condensed matter system and they are also expected to have appeared in phase transitions in the early universe as well (for a general review, please see [7]).

In the study of phase transitions in quantum field theory one basic quantity usually computed is the effective potential, which is an important tool in the study of phase transitions in scalar and gauge field theories [8]. It is equivalent to the homogeneous coarse-grained free-energy density functional of statistical physics, with its minima giving the stable and, when applicable, metastable states of the system. For interacting field theories the effective potential is evaluated perturbatively, with an expansion in loops being equivalent to an expansion in powers of $\hbar$ [9]. The one-loop approximation is then equivalent to incorporating the first quantum corrections to the classical potential. Recall that the effective potential, taking a scalar field theory as an example, is obtained from the effective action $\Gamma\left[\phi_{c}\right]$, where it is defined in terms of the connected generating functional $W[J]$ as

$$
\Gamma\left[\phi_{c}\right]=W[J]-\int d^{4} x J(x) \phi_{c}(x)
$$

with the classical field $\phi_{c}(\vec{x}, t)$ defined by $\phi_{c}(\vec{x}, t) \equiv$ $\delta W[J] / \delta J(x)$, and

$$
W[J]=-i \hbar \ln \int D \phi \exp \left[\frac{i}{\hbar} S[\phi, J]\right]
$$

In order to evaluate $\Gamma\left[\phi_{c}\right]$ perturbatively, one writes the field as $\phi(\vec{x}, t) \rightarrow \phi_{0}(\vec{x}, t)+\eta(\vec{x}, t)$, where $\phi_{0}(\vec{x}, t)$ is a field configuration which extremizes the classical action $S[\phi, J]$, $\left.\frac{\delta S[\phi, J]}{\delta \phi}\right|_{\phi=\phi_{0}}=0$, and $\eta(\vec{x}, t)$ is a small perturbation about that extremum configuration. The action $S[\phi, J]$ can then be expanded about $\phi_{0}(\vec{x}, t)$ and, up to quadratic order in $\eta(\vec{x}, t)$, we can use a saddle-point approximation to the path integral to obtain for the connected generating functional,

$$
W[J]=S\left[\phi_{0}\right]+\hbar \int d^{4} x \phi_{0}(x) J(x)+i \frac{\hbar}{2} \operatorname{Tr} \ln \left[\partial_{\mu} \partial^{\mu}+V^{\prime \prime}\left(\phi_{0}\right)\right] .
$$

In order to obtain the one-loop expression for $\Gamma\left[\phi_{c}\right]$, we first note that writing $\phi_{0}=\phi_{c}-\eta$ we get to first order in $\hbar, S\left[\phi_{0}\right]=$ $S\left[\phi_{c}\right]-\hbar \int d^{4} x \eta(x) J(x)+O\left(\hbar^{2}\right)$. Using this result and Eq. (3) into Eq. (1) we find, as $J \rightarrow 0$,

$$
\Gamma\left[\phi_{c}\right]=S\left[\phi_{c}\right]+i \frac{\hbar}{2} \operatorname{Tr} \ln \left[\partial_{\mu} \partial^{\mu}+V^{\prime \prime}\left(\phi_{c}\right)\right] .
$$

The effective action can also be computed as a derivative expansion about $\phi_{c}(\vec{x}, t)$,

$$
\Gamma\left[\phi_{c}\right]=\int d^{4} x\left[-V_{\mathrm{eff}}\left(\phi_{c}(x)\right)+\frac{1}{2}\left(\partial_{\mu} \phi_{c}\right)^{2} Z\left(\phi_{c}(x)\right)+\ldots\right]
$$


The function $V_{\text {eff }}\left(\phi_{c}\right)$ is the effective potential. For a constant field configuration $\phi_{c}(\vec{x}, t)=\phi_{c}$ we obtain

$$
\Gamma\left[\phi_{c}\right]=-\Omega V_{\mathrm{eff}}\left(\phi_{c}\right),
$$

where $\Omega$ is the total volume of space-time. Comparing Eqs. (4) and (6) we obtain for the one-loop effective potential,

$$
V_{\mathrm{eff}}\left(\phi_{c}\right)=V\left(\phi_{c}\right)-i \frac{\hbar}{2} \Omega^{-1} \operatorname{Tr} \ln \left[\partial_{\mu} \partial^{\mu}+V^{\prime \prime}\left(\phi_{c}\right)\right] .
$$

When working at non-vanishing temperature, the same functional techniques can be used. In this case one is interested in evaluating the generating functional (the partition function) $Z_{\beta}[J]$ which is given by the path integral [10]

$$
Z_{\beta}[J]=N \int D \phi \exp \left[-\int_{0}^{\beta} d \tau \int d^{3} x\left(\mathcal{L}_{E}-J \phi\right)\right]
$$

where the integration is restricted to paths periodic in $\tau$ with $\phi(0, \vec{x})=\phi(\beta, \vec{x}), \mathcal{L}_{E}$ is the Euclidean Lagrangian, and $N$ is a normalization constant. Again one expands about an extremum of the Euclidean action and calculates the partition function by a saddle-point evaluation of the path integral. The result for the one-loop approximation to the effective potential is

$$
\begin{aligned}
& V_{\mathrm{eff}}\left(\phi_{c}, T\right)=V_{\mathrm{eff}}\left(\phi_{c}\right) \\
& \quad+\frac{\hbar}{2 \pi^{2} \beta^{4}} \int_{0}^{\infty} d x x^{2} \ln \left\{1-\exp \left[-\sqrt{x^{2}+\beta^{2} V^{\prime \prime}\left(\phi_{c}\right)}\right]\right\}
\end{aligned}
$$

As the effective potential is equivalent to the free energy functional (for a constant field configuration), all thermodynamics functions follow from it. In particular the different phases, critical temperature of phase transition and temperature dependence of the field vacuum expectation value can be obtained from (9).

From the above discussion it is clear that the one-loop approximation to the effective action, Eq. (4), works best when the classical field does not differ much from the configuration that extremizes the classical action, $\phi_{c}=\phi_{0}+\eta \sim \phi_{0}$, since in this case the saddle-point evaluation to the path integral is adequate. Also, $\phi_{c}(\vec{x}, t)$ must be nearly constant so that the effective potential can be obtained from Eq. (6). As $J \rightarrow 0, \phi_{c}(\vec{x}, t)$ is identified with $\langle\phi\rangle$, the vacuum expectation value. One major problem we see in this whole approach of studying the phase structure of field theory models from the effective potential is when the action functional, determined from Eq. (8) is dominated not by homogeneous, constant field configurations but by nonhomogeneous ones. In those situations when other stable, finite energy solutions to the field equations of motion exist, we expect these configurations to dominate the partition function over the homogenous solutions for instance close to the critical temperature [11]. Under these circumstances the effective potential, which includes only contributions to the partition function from constant background field configurations becomes inappropriate to study the phase transition and we must rely in other approaches, for example studying the phase transition directly from the effective action or free energy for the topological configurations [11, 12], or taking directly a field theoretic description for the topological excitations [13]. In either case we are faced with the problem of accounting for nonlocal contributions in the perturbative expansion, which is only amenable of analysis, in most cases, up to the lowest leading order. To circumvent these difficulties we here will make use of the techniques of dualization in field theory, from which the degrees of freedom of the topological excitations are explicitly realized in the functional action. This method is described in the following sections. In Sec. II, we briefly review the model we will be using in this work, the scalar Abelian Higgs model, whose topological solutions are vortex-strings. In Sec. III we evaluated the dual action for the model, making explicit the vortex-strings degrees of freedom and how they couple to the matter fields. In Sec. IV we show how an effective potential calculation for an averaged vortex-string field can be computed and we discuss the results and interpretation of the phase transition obtained from this quantity. Finally, we give our conclusions in Sec. V.

\section{THE STRING SOLUTIONS IN THE SCALAR ABELIAN HIGGS MODEL}

In this work we will use the Abelian Higgs model, with Lagrangian density for a complex scalar field $\phi$ and gauge field $A_{\mu}$ given by

$$
\mathcal{L}=-\frac{1}{4} F_{\mu v} F^{\mu v}+\left|D_{\mu} \phi\right|^{2}-V(\phi)
$$

where, $F_{\mu v}=\partial_{\mu} A_{v}-\partial_{v} A_{\mu}, D_{\mu}=\partial_{\mu}-i e A_{\mu}$ and $V(\phi)$ is a symmetry breaking potential, for example given by

$$
V(\phi)=-m_{\phi}^{2}|\phi|^{2}+\frac{\lambda}{3 !}\left(|\phi|^{2}\right)^{2},
$$

with positive parameters $m_{\phi}^{2}$ and $\lambda$. The symmetry breaking $U(1) \rightarrow 1$ with homotopy group $\pi_{1} \neq 1$ indicates the existence of string-like topological excitations in the system, or Nielsen-Olesen strings [4] (for an extended introduction and review see e.g. Ref. [3]). For example, for a unit winding string solution along the $z$ axis, the classical field equations of motion obtained from the Lagrangian density (10) admit a stable finite energy configuration describing the string given by (using the cylindrical coordinates $r, \theta, z$ )

$$
\begin{aligned}
\phi_{\text {string }} & =\frac{\rho(r)}{\sqrt{2}} e^{i \theta}, \\
A_{\mu, \text { string }} & =\frac{1}{e} A(r) \partial_{\mu} \theta,
\end{aligned}
$$

where the functions $\rho(r)$ and $A(r)$ vanish at the origin and have the asymptotic behavior 


$$
\begin{aligned}
& \phi(r \rightarrow \infty) \rightarrow \rho_{v} \equiv \sqrt{\frac{6 m_{\phi}^{2}}{\lambda}}, \\
& A(r \rightarrow \infty) \rightarrow 1 .
\end{aligned}
$$

The functions $\rho(r)$ and $A(r)$ can be obtained numerically by solving the classical field equations for $\phi$ and $A_{\mu}$. If we write the field $\phi$ as $\phi=\rho \exp (i \chi) / \sqrt{2}$, then from (12) and (13) for the string, at spatial infinity $\rho$ goes to the vacuum $\rho_{v}$ and $A_{\mu}$ becomes a pure gauge. This also gives, in order to get a finite energy for the string configuration, that $\partial_{\mu} \chi=e A_{\mu}$ at $r \rightarrow \infty$, so $D_{\mu} \phi=0$. This leads then that, by taking some contour $C$ surrounding the symmetry axis, and using Stokes' theorem, that the magnetic flux is nonvanishing,

$$
\Phi=\oint A_{\mu} d x^{\mu}=\oint \partial_{\mu} \chi d x^{\mu}=2 \pi / e .
$$

Since $\phi$ must be single-valued, the Eq. (15) implies that on the string $\chi$ must be singular. Therefore, the phase $\chi$ can be separated into two parts: in a regular and in a singular part, due to the string configuration,

$$
\chi(x)=\chi_{\mathrm{reg}}(x)+\chi_{\operatorname{sing}}(x),
$$

where the singular (multivalued) part $\chi_{\text {sing }}(x)$ can be related to a closed world-sheet of a vortex-string [14],

$$
\frac{1}{2 \pi} \varepsilon_{\mu \nu \lambda \rho} \partial_{\lambda} \partial_{\rho} \chi_{\text {sing }}(x)=n \int_{S} d \sigma_{\mu v}(x) \delta^{4}[x-y(\xi)]=\omega_{\mu v},
$$

where $n$ is a topological quantum number, the winding number, which we here restrict to the lowest values, $n= \pm 1$, corresponding to the energetically dominant configurations. The element of area on the world sheet swept by the string is given by

$$
d \sigma_{\mu v}(x)=\left(\frac{\partial x_{\mu}}{\partial \xi^{0}} \frac{\partial x_{v}}{\partial \xi^{1}}-\frac{\partial x_{\mu}}{\partial \xi^{1}} \frac{\partial x_{v}}{\partial \xi^{0}}\right) d^{2} \xi
$$

and $y_{\mu}(\xi)$ represents a point on the world sheet $S$ of the vortexstring, with internal coordinates $\xi^{0}$ and $\xi^{1}$. As usual, we consider that $\xi^{1}$ is a periodic variable (we work here with closed strings), whereas $\xi^{0}$ will be proportional to the time variable (at zero temperature), in such a way that $\xi^{1}$ parameterizes a closed string at a given instant $\xi^{0}$. Eq. (17) is known as the vorticity. Eqs. (17) and (18) entails the vortex-string degrees of freedom and then can be used to identify the topological vortex string contributions to the partition function.

Let us briefly recall two main previous methods that try to take into account the effect of topological strings in phase transitions. The first attempt to do so made use of semiclassical methods [2]. In the semiclassical method we use directly the nonhomogeneous string solutions, Eqs. (12) and (13), when evaluating the effective action. In this case the effective action is evaluated after taking fluctuations around the string vacuum solutions, $\phi \rightarrow \phi_{\text {string }}+\phi^{\prime}$ and $A_{\mu} \rightarrow A_{\mu \text {,string }}+A_{\mu}^{\prime}$ and the functional integration performed over the fluctuation fields $\phi^{\prime}$ and $A_{\mu}^{\prime}$. In the one-loop approximation, this gives the analogous to Eq. (4), with the constant background field now replaced by the scalar string background configuration plus those analogous loop corrections for the gauge field string configuration. But from Eqs. (4) and (8), we see that the effective action at finite temperature is just associated with the free energy of the system, where, here is the free energy in the presence of the string field configurations. This is the procedure used for instance in the papers in Ref. [11]. The free energy relevant for the study is written as [11]

$$
F_{\text {string }}=-\frac{1}{\beta L} \ln \left(\frac{Z_{\text {string }}}{Z_{v}}\right) \text {, }
$$

where $Z_{\text {string }}$ is the partition function evaluated in the presence (imposing the appropriate boundary conditions for) of strings, while $Z_{v}$ is the partition function for the trivial (constant) vacuum sector of the model (and then Eq. (19) is actually the free energy difference between the string and trivial vacuum sectors). $\beta$, as always, is the inverse of the temperature (we use throughout this work, unless explicitly noted, the natural units $\left.\hbar, c, k_{B}=1\right)$ and $L$ is the size of the system.

The difficult with the approach given by (19), which becomes evident from Eq. (4) when we are dealing with non constant background fields, is the nonlocal terms that appears in higher order perturbation terms when expanding the effective action (in this case, the effective action for the string background configurations). The only terms amenable of analysis are the one-loop leading order terms. Analogous approach based on the semiclassical method, is the direct evaluation of the classical partition function taking into account the string degrees of freedom, as performed by the authors in Ref. [12] and where the contribution and interpretation of the phase transition based on the picture of string condensation is analyzed using known statistical physics results.

Another approach that has been used is to define a field creation operator for vortex-string excitations and then work directly with the correlation functions in terms of these operators. This is the approach for instance taken in Refs. [13, 15]. However, also in this approach the evaluation of correlation functions already at tree-level order is involved and results are lacking beyond that order (though in the first reference of [13] results for the asymptotic behavior of the two-point correlation function for vortex operators were obtained at one-loop order, but only for the $2+1$ dimensions case).

\section{THE DUAL ACTION FOR VORTEX-STRINGS}

Let us start by writing the partition function for the Abelian Higgs model (10), which, in Euclidean space-time is given by

$$
Z_{\beta}=\int \mathcal{D} A \mathcal{D} \phi \mathcal{D} \phi^{*} \exp \left\{-\int_{0}^{\beta} d \tau \int d^{3} x \mathcal{L}_{E}-S_{G F}\right\}
$$


where in the above expression $\mathcal{L}_{E}$ denotes the Lagrangian density (10) in Euclidian space-time and $S_{G F}$ is some appropriate gauge-fixing and ghost term that must be added to the action to perform the functional integral over the relevant degrees of freedom. A dual action to the original one is obtained from (20) by appropriately performing Hubbard-Stratonovich transformations on the original field in such a way to become explicitly the strings degrees of freedom, like in the form of Eq. (16) and (17). For that, we first write the complex Higgs field $\phi$ in the polar parameterization form $\phi=\rho e^{i \chi} / \sqrt{2}$. Then, the scalar phase field $\chi$ is split in its regular and singular terms, like in Eq. (16).

Lets for now, for convenience, omit the gauge fixing term $S_{G F}$ in Eq. (20) and re-introduce it again in the final transformed action. Following e.g. the procedure of Refs. [16-20], the functional integral over $\chi$ in Eq. (20) can then be rewritten as

$$
\begin{aligned}
& \int \mathcal{D} \chi \exp \left[-\int d^{4} x \frac{1}{2} \rho^{2}\left(\partial_{\mu} \chi+e A_{\mu}\right)^{2}\right] \\
& =\int \mathcal{D} \chi_{\text {sing }} \mathcal{D} \chi_{\text {reg }} \mathcal{D} C_{\mu}\left(\prod_{x} \rho^{-4}\right) \exp \left\{-\int d^{4} x\left[\frac{1}{2 \rho^{2}} C_{\mu}^{2}-i C_{\mu}\left(\partial_{\mu} \chi_{\mathrm{reg}}\right)-i C_{\mu}\left(\partial_{\mu} \chi_{\text {sing }}+e A_{\mu}\right)\right]\right\} \\
& =\int \mathcal{D} \chi_{\text {sing }}\left(\prod_{x} \rho^{-4}\right) \mathcal{D} W_{\mu \nu} \exp \left\{-\int d^{4} x\left[\frac{\kappa^{2}}{2 \rho^{2}} V_{\mu}^{2}+e \kappa A_{\mu} V_{\mu}+i \pi \kappa W_{\mu v} \omega_{\mu v}\right]\right\},
\end{aligned}
$$

where we have performed the functional integral over $\chi_{\text {reg }}$ in the second line of Eq. (21). This gives a constraint on the functional integral measure, $\delta\left(\partial_{\mu} C_{\mu}\right)$, which can be represented in a unique way by expressing the $C_{\mu}$ in terms of an antisymmetric field, $C_{\mu}=-i \frac{\kappa}{2} \varepsilon_{\mu \nu \lambda \rho} \partial_{\nu} W_{\lambda \rho} \equiv \kappa V_{\mu}$, which then leads to the last expression in Eq. (21). $\kappa$ is some arbitrary parameter with mass dimension and $\omega_{\mu v}$ is the vorticity, defined by Eq. (17) for the singular phase part of $\chi$. Next, in order to linearize the dependence on the gauge field in the action we introduce a new antisymmetric tensor field $G_{\mu \nu}$ through the identity

$$
\begin{aligned}
& \exp \left(-\frac{1}{4} \int d^{4} x F_{\mu v}^{2}\right) \\
& =\int \mathcal{D} G_{\mu v} \exp \left[\int d^{4} x\left(-\frac{\mu_{W}^{2}}{4} G_{\mu \nu}^{2}-\frac{\mu_{W}}{2} \tilde{G}_{\mu v} F_{\mu v}\right)\right]
\end{aligned}
$$

with $\tilde{G}_{\mu \nu} \equiv \frac{1}{2} \varepsilon_{\mu \nu \lambda \rho} G_{\lambda \rho}$.
Substituting Eqs. (21) and (22) back into the partition function, we can immediately perform the functional integral over the $A_{\mu}$ field. This leads to the constraint $\varepsilon_{\mu v \alpha \beta} \partial_{\mu}\left(G_{\alpha \beta}-W_{\alpha \beta}\right)=0$, which can be solved by setting $G_{\mu \nu}=W_{\mu \nu}-\frac{1}{\mu_{W}}\left(\partial_{\mu} B_{v}-\partial_{v} B_{\mu}\right)$, where we have defined, for convenience, $e \kappa=\mu_{W}$ and $B_{\mu}$ is an arbitrary gauge field. Using these expressions back in the partition function (and reintroducing the gauge fixing term) we then finally obtain the result

$$
\begin{aligned}
Z & =\int \mathcal{D} W_{\mu v} \mathcal{D} \chi_{\text {sing }} \mathcal{D} B_{\mu} \mathcal{D} \rho\left(\prod_{x} \rho^{-3}\right) \\
& \times \exp \left\{-S_{\text {dual }}\left[W_{\mu v}, B_{\mu}, \rho, \chi_{\text {sing }}\right]-S_{G F}\right\}
\end{aligned}
$$

where the dual action is given by

$$
S_{\text {dual }}=\int d^{4} x\left[\frac{\mu_{W}^{2}}{2 e^{2} \rho^{2}} V_{\mu}^{2}+\frac{1}{4}\left(\mu_{W} W_{\mu v}-\partial_{\mu} B_{v}+\partial_{v} B_{\mu}\right)^{2}+\frac{1}{2}\left(\partial_{\mu} \rho\right)^{2}-\frac{m_{\phi}^{2}}{2} \rho^{2}+\frac{\lambda}{4 !} \rho^{4}+i \pi \frac{\mu_{W}}{e} W_{\mu v} \omega_{\mu v}\right] \text {. }
$$

The model described by $S_{\text {dual }}$ is completely equivalent to the original Abelian Higgs model, in the polar representation obtained from Eq. (20) and so, any calculations done using (23) must lead to the same results as those done with the original action. The advantage of this dual formulation (24) is that it explicitly exhibits the dependence on the singular configura- tion of the Higgs field, making it appropriate to study phase transitions driven by topological defects. At the same time it also shows, from the last term in Eq. (24), that the vortexstring's degrees of freedom are coupled to the matter field through the antisymmetric (or Kalb-Ramond) field. Now, if we come to the part concerning the gauge fixing term $S_{G F}$ in 
(23), we see from Eq. (24) that the dual action exhibits invariance under the double gauge transformation: the hypergauge transformation

$$
\begin{aligned}
\delta W_{\mu v}(x) & =\partial_{\mu} \xi_{v}(x)-\partial_{v} \xi_{\mu}(x), \\
\delta B_{\mu} & =\mu_{W} \xi_{\mu}(x),
\end{aligned}
$$

and the usual gauge transformation

$$
\delta B_{\mu}=\partial_{\mu} \theta(x),
$$

where $\xi_{\mu}(x)$ and $\theta(x)$ are arbitrary vector and scalar functions, respectively. Choosing $\xi_{\mu}=B_{\mu}$ in the first transformation is equivalent to fix the gauge through the condition $B_{\mu}=0$ [17] and this is equivalent to choose the unitary gauge in Eq. (23). The complete form for the gauge fixing action accounting for the gauge invariances (25) and (26) was obtained in Ref. [21], which, besides an overall normalization factor independent of the action fields (and the background Higgs field) gives for the quantum partition function the complete result [21]

$$
\begin{aligned}
Z & =N \int \mathcal{D} W_{\mu v} \mathcal{D} \rho \mathcal{D} B_{\mu} \mathcal{D} \bar{\eta} \mathcal{D} \eta \exp \left\{-\int d^{4} x\left[\frac{\mu_{W}^{2}}{2 e^{2} \rho^{2}} V_{\mu}^{2}+\frac{1}{4}\left(\mu_{W} W_{\mu v}-\partial_{\mu} B_{v}+\partial_{v} B_{\mu}\right)^{2}\right.\right. \\
& \left.\left.+\frac{1}{2}\left(\partial_{\mu} \rho\right)^{2}-\frac{m_{\phi}^{2}}{2} \rho^{2}+\frac{\lambda}{4 !} \rho^{4}-\bar{\eta} \rho^{-3} \eta-\frac{1}{2 \theta}\left(\partial^{\mu} W_{\mu v}\right)^{2}+\frac{u}{2 \theta} \mu_{W} W_{\mu v}\left(\partial^{\mu} B^{v}-\partial^{v} B^{\mu}\right)+\frac{1}{2 \xi}\left(\partial_{\mu} B^{\mu}\right)^{2}\right]\right\}
\end{aligned}
$$

where $\bar{\eta}, \eta$ are the ghost fields used to exponentiate the Jacobian $\rho^{-3}$ in the functional integration measure in Eq. (23) and $\theta, u$ and $\xi$ are gauge parameters.

\section{THE EFFECTIVE POTENTIAL FOR LOCAL VORTEX-STRINGS AVERAGED FIELDS}

Let us turn now to the study of the problem of vortex-strings condensation during a phase transition. Thus, to proceed further with the evaluation of the string contribution to the partition function we introduce a (nonlocal) field associated to the string. Quantizing the vortex-strings as nonlocal objects and associating to them a wave function $\Psi[C]$, a functional field, where $C$ is the closed vortex-string curve in Euclidean space-time, and noting that the interaction term of the vortexstring with the antisymmetric field in Eq. (24) is in the form of a current coupled to the antisymmetric field, following Refs. $[22,23]$ we can define the string action term in the form

$$
S_{\text {string }}\left(\Psi[C], W_{\mu \nu}\right)=\oint_{C} d x_{v}\left[\left|D_{\sigma^{\mu \nu}} \Psi[C]\right|^{2}-M_{0}^{2}|\Psi[C]|^{2}\right],
$$

where $D_{\sigma^{\mu v}}$ is a covariant derivative term defined by [24]

$$
D_{\sigma^{\mu v}}(x)=\frac{\delta}{\delta \sigma^{\mu v}(x)}-i \frac{2 \pi \mu_{W}}{e} W_{\mu v}(x)
$$

where $\delta \sigma^{\mu v}(x)$ is to be considered as an infinitesimal rectangular deformation on the string's worldsheet. It can be easily checked that Eq. (28) is invariant under the combined gauge invariances (25) and (30) if the hypergauge transformation (25) is now supplemented by the vortex-string field transformation

$$
\Psi[C] \rightarrow \exp \left[-i \frac{2 \pi \mu_{W}}{e} \oint d x^{\mu} \xi_{\mu}(x)\right] \Psi[C] .
$$

$M_{0}^{4}$ in Eq. (28) is a dynamical mass for the strings,

$$
M_{0}^{4} \equiv \frac{1}{a^{4}}\left(e^{\tau_{s} a^{2}}-6\right)
$$

with $\tau_{s}$ is the string tension (the total energy per unit length of the vortex-string) [22, 23], which, in terms of the parameters of the Abelian Higgs model, it is given by [25] $\tau_{s}=\pi \rho_{c}^{2} \varepsilon\left(\lambda / e^{2}\right)$, where $\varepsilon\left(\lambda / e^{2}\right)$ is a function that increases monotonically with the ratio of coupling constants. $a$ in Eq. (31) can approximately be given by the string typical radius and be expressed as [12]

$$
1 / a \sim m_{\phi}\left(1-\frac{T^{2}}{T_{c}^{2}}\right)^{1 / 2},
$$

where $T_{c}$ is the mean-field critical temperature, $T_{c}=$ $\sqrt{12 m_{\phi}^{2} /\left(3 e^{2}+2 \lambda / 3\right)}[26] . \rho_{c}$, the Higgs vacuum expectation value, can likewise be expressed as

$$
\rho_{c} \simeq \sqrt{\frac{6 m_{\phi}^{2}}{\lambda}}\left(1-\frac{T^{2}}{T_{c}^{2}}\right)^{1 / 2} .
$$

By defining a local string field as [22]

$$
\hat{\psi}_{C} \equiv 4\left(\frac{2 \pi}{e}\right)^{2} \sum_{C_{x, t}} \frac{1}{a^{3} l}|\Psi[C]|^{2}
$$


where $l$ is the length of a curve $C$, and $C_{x, t}$ represents a curve passing through a point $x$ in a fixed direction $t$, the vacuum expectation value of $\hat{\psi}_{C}$, denoted by $\psi_{C}$, represents the sum of existence probabilities of vortices in $C_{x, t}$. In terms of $\hat{\psi}_{C}$, it can be shown that the contribution of the vortices to the quantum partition function, indicated by the last term in Eq. (24) and the integration over $\chi_{\text {sing }}$, can be written as [22]

$$
\int \mathcal{D} \Psi[C] \mathcal{D} \Psi^{*}[C] e^{-\int d^{4} x\left[\frac{1}{4}\left(\frac{e}{2 \pi}\right)^{2} M_{0}^{4} \hat{\psi}_{C}+\frac{\mu_{W}^{2}}{4} W_{\mu v}^{2} \hat{\Psi}_{C}\right]} .
$$

Eq. (35) implies, together with Eq. (24), that an immediate consequence of $\psi_{C} \neq 0$ is the increase of the $W_{\mu v}$ mass. This is directly associated with a shift in the mass of the original gauge field in the broken phase, $M_{A}=e \rho_{c}$, as $M_{A}^{2} \rightarrow M_{A}^{2}\left(1+\psi_{C}\right)$. Since the field $\psi_{C}$, defined by Eq. (34), works just like a local field for the vortex-strings, we are allowed to define an effective potential for its vacuum expectation value $\psi_{C}$ in just the same way as we do for a constant Higgs field. Since this vortex-string field only couples directly to $W_{\mu v}$, at the one-loop level the effective potential for $\psi_{C}$ will only involve internal propagators of the antisymmetric tensor field. This effective potential, at one-loop order and at $T=0$, was actually computed in Ref. [22] in the Landau gauge for the antisymmetric tensor field propagator. Its generalization to finite temperatures is immediate and it gives

$$
\begin{aligned}
& V_{\text {eff }}^{1-\text { loop }}\left(\psi_{C}\right)=\frac{e^{2}}{4 \pi^{2}} M_{0}^{4} \psi_{C} \\
& \quad+\frac{3}{2} \frac{1}{\beta} \sum_{n=-\infty}^{+\infty} \int \frac{d^{3} k}{(2 \pi)^{3}} \ln \left[\omega_{n}^{2}+\mathbf{k}^{2}+M_{A}^{2}\left(1+\psi_{C}\right)\right]
\end{aligned}
$$

where $\omega_{n}=2 \pi n / \beta$ are the Matsubara frequencies for bosons. When $\psi_{C}=0$, in the absence of string vacuum contributions to the partition function, we re-obtain the standard result for the one-loop contribution to the Higgs effective potential coming from the gauge field loops. The sum over the Matsubara frequencies in (36) is easily performed [26]. We can also work with the resulting expression by expanding it in the high-temperature limit $M_{A} \sqrt{1+\psi_{C}} / T \ll 1$ and for $e^{2} / \lambda \ll 1$, which corresponds to deep in the second order regime of phase transition for the scalar Abelian Higgs model. This is analogous to the phenomenology of the Landau-Ginzburg theory for superconductors, where the parameter $\left(e^{2} / \lambda\right)^{-1}$ (also called the Ginzburg parameter), measuring the ratio of the penetration depth and the coherence length, controls the regimes called Type II and Type I superconductors. In our case, the coherence length is governed by $a \sim 1 / M_{H}$, where $M_{H}$ is here the temperature dependent Higgs mass, while the penetration depth is proportional to $1 / M_{A}$, where $M_{A}$ is the (temperature dependent) gauge field mass. This way we find a manageable expression for the finite temperature effective potential given by [21]

$$
\begin{gathered}
V_{\text {eff,string }}^{(\beta)}\left(\psi_{C}\right) \simeq\left[\frac{e^{2}}{4 \pi^{2}} M_{0}^{4}+\frac{3 e^{2} \rho_{c}^{2}}{16 \pi^{2} a^{2}}+\frac{e^{2} \rho_{c}^{2}}{8} T^{2}\right] \psi_{C} \\
-\frac{e^{3} \rho_{c}^{3}}{4 \pi}\left(1+\psi_{C}\right)^{3 / 2} T-\frac{3 e^{4} \rho_{c}^{4} \ln [2 / a T]}{32 \pi^{2}} \psi_{C}^{2},
\end{gathered}
$$

where $M_{0}, a$ and $\rho_{c}$ are given by Eqs. (31), (32) and (33).

We can then see that the quantum and thermal corrections in the effective potential for strings, Eq. (37), are naturally ordered in powers of $\alpha=e^{2} / \lambda$. Therefore, in the regime $\alpha \ll 1$ the leading order correction to the tree-level potential in Eq. (37) is linear in $\psi_{C}$, while the second and the third correction terms are $O\left(\alpha^{3 / 2}\right)$ and $O\left(\alpha^{2}\right)$, respectively. Thus, the linear term in $\psi_{C}$ controls the transition in the deep second order regime since the other terms are all subleading in $\alpha$. Thus, near criticality, determined by some temperature $T_{S}$ where the linear term in Eq. (37) vanishes, $V_{\text {eff,string }}^{(\beta)}\left(\psi_{C}\right) \sim 0$ in the $\alpha \ll 1$ regime. $T_{s}$ is interpreted as the temperature of transition from the normal vacuum to the state of condensed strings and it is then determined by the temperature where the linear term in $\psi_{C}$ in Eq. (37) vanishes and it is found to be related to the mean field critical temperature, for which the effective mass term of the Higgs field, obtained from $V_{\text {eff }}^{(\beta)}\left(\rho_{c}\right)$, vanishes. Using again Eqs. (32) and (33), with the result $\tau_{s} a^{2} \sim O(1 / \lambda)$ and in the perturbative regime $e^{2} \ll \lambda \ll 1$, after some straightforward algebra, we find the relation

$$
\frac{T_{c}-T_{s}}{T_{c}} \sim O\left(\frac{e^{-1 / \lambda}}{\lambda^{2}}\right)[1+O(\alpha)],
$$

with next order corrections to the critical temperatures difference being of order $O(\alpha)$. This result for $T_{s}$ allows us to identify it with the Ginzburg temperature $T_{G}$ for which the contribution of the gauge field fluctuations become important. These results are also found to be in agreement with the calculations done by the authors in Ref. [12], who analyzed an analogous problem using the partition function for strings configurations, in the same regime of deep second order transition.

For the case where the gauge fluctuations are stronger, i.e., for $\alpha=e^{2} / \lambda \gtrsim 1$, the second term in Eq. (37) of order $\alpha^{3 / 2}$ induces a cubic term $\rho_{c}^{3}$ to the effective potential, favoring the appearance of a first order phase transition instead of a second order one. Here we see that the non-trivial vacuum $\psi_{c} \neq 0$ above the critical temperature $T_{s}$ enhances the first order phase transition by an amount $\left(1+\psi_{c}\right)^{3 / 2}$. Hence, since $T_{S} \sim T_{c}$, we see that the driven mechanism of the first order transition can be interpreted as a melting of topological defects. This mechanism is very well known in condensed matter physics [20] and always leads to a first order phase transition (except in two dimensions).

\section{CONCLUSIONS}

We have interpreted here the phase transition in the scalar Abelian Higgs model as a process of condensation of vortexstrings. Our analysis was based on a dual realization of the 
original model in such a way to make explicit the vortexstrings degrees of freedom of the nontrivial vacuum of the model. This way, by constructing a field theory model for string fields, the finite temperature effective potential for a local expectation value for the string field was obtained. The transition temperature obtained from this effective potential, the temperature of transition from the normal vacuum to the state of condensed strings, was then obtained and identified with the Ginzburg temperature for which gauge field fluctuations become important.

Possible extensions of this work could, for example, include magnetic monopoles, like in the context of the compact Abelian Higgs model [27], in which case monopoles could be added as external fields in the dual transformations. The study of finite temperature effects and possible consequences for the confinement picture in the dual superconductor model, should be possible in the context of the study performed in this work and it is an interesting venue for future work.

\section{Acknowledgments}

The authors would like to thank Conselho Nacional de Desenvolvimento Científico e Tecnológico (CNPq-Brazil), Fundação de Amparo à Pesquisa do Estado do Rio de Janeiro (FAPERJ) for the financial support. R.O.R. would like to thank the organizers of the conference Infrared QCD in Rio for the invitation to talk about this work at the conference.
[1] S. Coleman, Aspects of Symmetry (Cambridge University Press, Cambridge, 1985).

[2] R. Rajaraman, Solitons and Instantons (North Holland, Amsterdam, 1989).

[3] A. Vilenkin and E. P. S. Shellard, Cosmic Strings and Other Topological Defects (Cambridge University Press, Cambridge, 2000).

[4] H. B. Nielsen and P. Olesen, Nuc. Phys. B 61, 45 (1973).

[5] G. 't Hooft, Nucl. Phys. B 79, 276 (1974).

[6] A. M. Polyakov, JETP Lett. 20, 194 (1974); Soviet Phys. JETP 41, 988 (1976)

[7] T. W. B. Kibble, Symmetry breaking and defects, in T.W.B. Kibble (Imperial Coll., London) . IMPERIAL-02-3-5, Nov 2002. 35pp. Lectures given at NATO Advanced Study Institute and COSLAB School: Patterns of Symmetry Breaking, Crakow, Poland, 15-29 Sep 2002. ArXive: cond-mat/0211110.

[8] L. Dolan and R. Jackiw, Phys. Rev. D 9, 3320 (1974); S. Weinberg, Phys. Rev. D 9, 3357 (1974).

[9] S. Coleman and E. Weinberg, Phys. Rev. D 3, 1888 (1973); P. Ramond, Field Theory: A Modern Primer, (Addison-Wesley, 1990).

[10] J. Kapusta, Finite Temperature Field Theory, (Cambridge University Press, 1989).

[11] C. A. de Carvalho, D. Bazeia, O. J. P. Eboli, and G. C. Marques, Phys. Rev. D 32, 3256 (1985); J. M. Guerra Jr., G. C. Marques, and S. J. Rodrigues, Phys. Rev. D 42, 2022 (1990).

[12] E. Copeland, D. Haws, and R. Rivers, Nuc. Phys. B 319, 687 (1989).

[13] E. C. Marino, G. C. Marques, R. O. Ramos, and J. S. Ruiz,
Phys. Rev. D 45, 3690 (1992); E. C. Marino and R. O. Ramos, Phys. Rev. D 49, 1093 (1994).

[14] P. A. M. Dirac, Phys. Rev. D 74, 817 (1948).

[15] E. C. Marino, Phys. Rev. D 53, 1001 (1996).

[16] K. Lee, Phys. Rev. D 48, 2493 (1993).

[17] P. Orland, Nuc. Phys. B 428, 221 (1994).

[18] E. T. Akhmedov, M. N. Chernodub, M. I. Polikarpov, and M. A. Zubkov, Phys. Rev. D 53, 2087 (1996).

[19] D. Antonov and D. Ebert, Eur. Phys. J. C 12, 349 (2000); D. Antonov, Int. J. Mod. Phys. A 14, 4347 (1999).

[20] H. Kleinert, Lett. Nuovo Cimento 35, 405 (1982); Phys. Lett. B 293, 168 (1992); see also, Chapter 13 in the second volume of H. Kleinert, Gauge fields in Condensed Matter, Vol I: Superflow and Vortex lines (World Scientific, Singapore, 1989); Gauge fields in Condensed Matter, Vol II: Stresses and Defects, Differential geometry, Crystal defects (World Scientific, Singapore, 1989)

[21] R. O. Ramos, J. F. Medeiros Neto, D. G. Barci, and C. A. Linhares, Phys. Rev. D 72, 103524 (2005).

[22] K. Seo and A. Sugamoto, Phys. Rev. D 24, 1630 (1981).

[23] H. Kawai, Progr. Theor. Phys. 65, 351 (1981).

[24] Y. Nambu, in Quark confinement and field theory: proceedings (Wiley, NY, 1977); Phys. Rept. 23, 250 (1976).

[25] M. B. Hindmarsh and T. W. B. Kibble, Rept. Progr. Phys. 48, 477 (1995)

[26] L. Dolan and R. Jackiw, Phys. Rev. D 9, 2904 (1974).

[27] M. N. Chernodub, Phys. Rev. D 69, 094504 (2004). 